\title{
COMPOSITION AND GALACTIC CONFINEMENT \\ OF COSMIC RAYS
}

\author{
MAURICE M. SHAPIRO
}

Laboratory for Cosmic Ray Physics, Naval Research Laboratory, Washington, D.C. 20390, U.S.A.

\section{Introduction}

The 'Galactic' cosmic rays impinging on the Earth come from afar over tortuous paths, traveling for millions of years. These particles are the only known samples of matter that reach us from regions of space beyond the solar system. Their chemical and isotopic composition and their energy spectra provide clues to the nature of cosmic-ray sources, the properties of interstellar space, and the dynamics of the Galaxy. Various processes in high-energy astrophysics could be illuminated by a more complete understanding of the arriving cosmic rays, including the electrons and gamma rays.*

En route, some of the primordial ${ }^{* *}$ cosmic-ray nuclei have been transformed by collision with interstellar matter, and the composition is substantially modified by these collisions. A dramatic consequence of the transformations is the presence in the arriving 'beam' of considerable fluxes of purely secondary elements $(\mathrm{Li}, \mathrm{Be}, \mathrm{B})$, i.e., species that are, in all probability, essentially absent at the sources. We shall here discuss mainly the composition of the arriving 'heavy' nuclei - those heavier than helium - and what they teach us about the source composition, the galactic confinement of the particles, their path lengths, and their transit times.

\section{Composition in the Vicinity of the Earth}

The distribution in abundance of the cosmic ray elements arriving at the Earth has recently been summarized by Shapiro and Silberberg (1970b). ${ }^{\dagger}$ Tables I and II, adapted from their review, list abundances of elements $\mathrm{Li}$ to Si (relative to carbon), and $\mathrm{P}$ to $\mathrm{Fe}$ (relative to the iron group), respectively. In Table $\mathrm{I}$ the abundances are given for relativistic particles, and in Table II, for slower ones as well. Some idea of

\footnotetext{
* For recent reviews of electrons in the cosmic rays, see, e.g., P. Meyer (1969), and Daniel and Stephens (1970). For reviews of cosmic $\gamma$-rays, see, e.g., Fazio (1967), Garmire and Kraushaar (1965), Greisen (1966, 1970), Gould and Burbidge (1967), Lüst and Pinkau (1967), Duthic (1968), Clark et al. (1968), Ginzburg and Syrovatskii (1964), Hayakawa (1969), Clark (1970), Fichtel (1970), and Stecker (1971). Cosmic neutrinos are still elusive, but an imaginative attack on this problem has been launched (Crouch et al., 1970; Krishnaswamy et al., 1970).

** Because the term 'primary' has been widely applied to particles arriving at the top of the Earth's atmosphere, we shall refer to those starting out at the sources as 'primordial'.

$\dagger$ That review is considerably more comprehensive than the present report; it includes references to much of the literature on composition and related problems. Regrettably, the present paper cannot do comparable justice to many important contributions. It touches mainly upon highlights of NRL work and, especially, some results of extensive calculations carried out since 1967 in collaboration with R. Silberberg and C. H. Tsao.
} 


\section{TABLE I}

Abundances of cosmic-ray elements $\mathrm{Li}$ to $\mathrm{Si}$ (relative to carbon at the top of the atmosphere)

$\begin{array}{lcrc}Z & \begin{array}{l}\text { Relative } \\ \text { abundance }\end{array} & Z & \begin{array}{l}\text { Relative } \\ \text { abundance }\end{array} \\ 3 & 16 \pm 2^{\mathrm{a}} & 9 & 2 \pm 1 \\ 4 & 11 \pm 3^{\mathrm{a}} & 10 & 20 \pm 2 \\ 5 & 27 \pm 3^{\mathrm{a}} & 11 & 3 \pm 1.5 \\ 6 & 100^{\mathrm{b}} & 12 & 21 \pm 2 \\ 7 & 27 \pm 2 & 13 & 2 \pm 1 \\ 8 & 86 \pm 4 & 14 & 15 \pm 2 \\ \\ \text { a Provisional values based on assuming } \\ \text { Be/B=0.4 and } \mathrm{Be} / \mathrm{Li}=\mathbf{0 . 7 .} \text {. } \\ \text { b Normalization. }\end{array}$

the remaining uncertainties in the fluxes of elements heavier than silicon can be obtained by comparing the last two rows of Table II. Despite the different mean energies of the nuclei in the two cases, the particles are all relativistic; one would not expect the abundance values in the last two rows to differ by as much as they do, e.g., for $Z=17,19,20$, or 25 . There are, moreover, indications of systematic experimental differences between Lezniak et al. (1970) (the second row) and the other workers (third row): (a) the abundance ratio of the set $16 \leqslant Z<23$ to the set $24 \leqslant Z \leqslant 26$, is 0.87 in one case, and 0.57 in the other; (b) the odd-to-even- $Z$ ratios are generally greater in the former than in the latter.

Some puzzling discrepancies also remain even for the elements in Table I, for which relative abundances are fairly well established from carbon to silicon. This is particularly true of the internal distribution of abundances among the three light elements $\mathrm{Li}, \mathrm{Be}$, and $\mathrm{B}$, as we shall see below.

Figure 1 compares the chemical composition of the arriving cosmic ray species with that of the elements in the solar photosphere. Solar abundances were normalized to a value of $10^{12}$ for hydrogen; cosmic-ray data were normalized so that the carbon value coincided with that for solar carbon. (For $\mathrm{F}$ and $\mathrm{Cl}$, meteoritic values were, perforce, substituted for solar ones.) The relative under-abundance, by an order of magnitude, of cosmic-ray hydrogen and helium in the cosmic rays, and the anomalous over-abundance of $\mathrm{Li}, \mathrm{Be}$, and $\mathrm{B}$, by five orders of magnitude, are among the striking features of the distribution.

\section{Path Through Interstellar Matter}

The amount of interstellar material traversed by cosmic rays from their sites of origin to the earth is a crucial parameter for calculations of cosmic ray propagation. As we shall see, one actually needs the distribution of path lengths in order to deduce the transmutations of the primordial cosmic ray elements into the composition we 


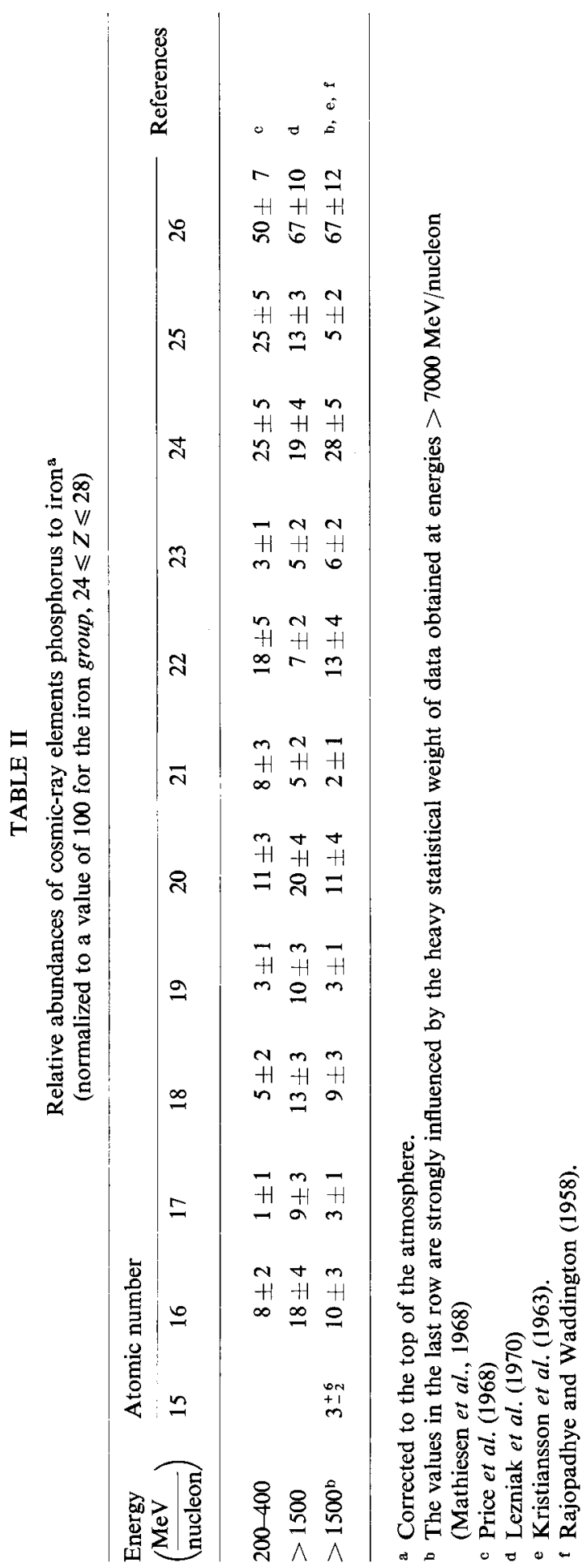




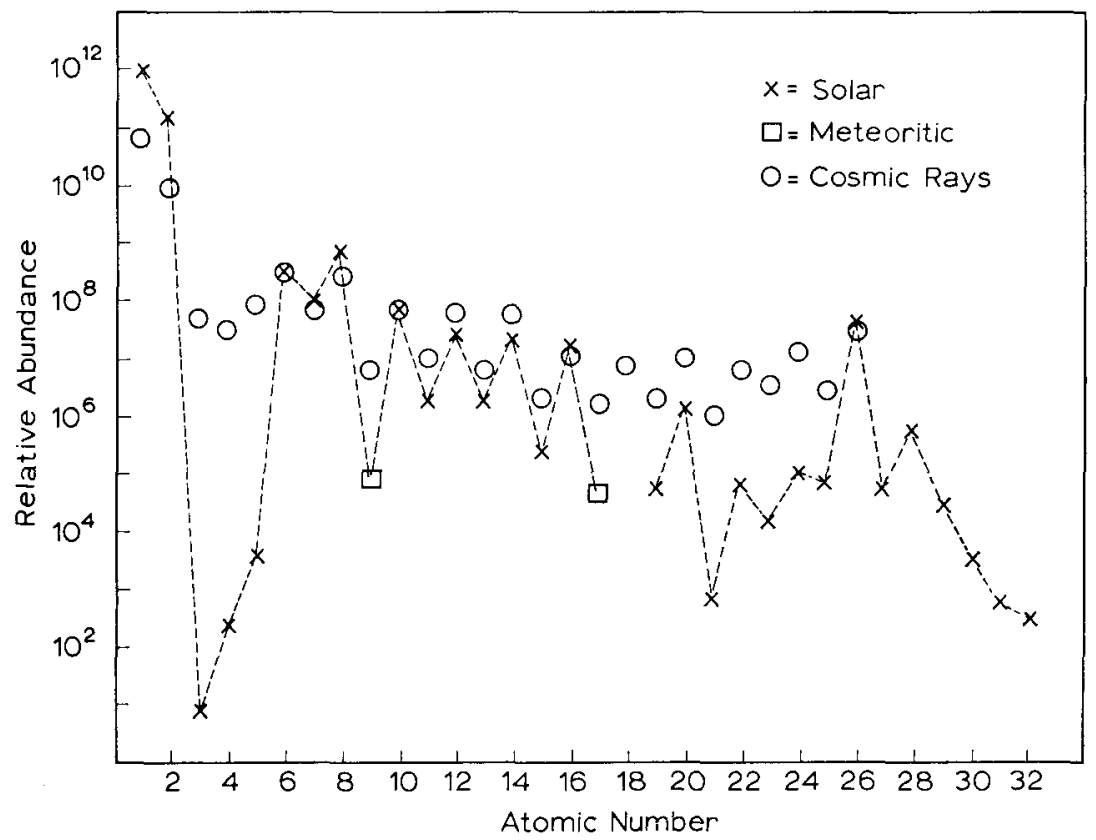

Fig. 1. Chemical composition of the arriving cosmic rays compared with that of the solar photosphere (for $\mathrm{F}$ and $\mathrm{C}$, the data are meteoritic). Solar abundances were normalized to a value of $10^{12}$ for hydrogen; cosmic-ray data were normalized so that the carbon value coincided with that of solar carbon.

observe near the earth. Conversely, certain features of this arriving composition enable us to arrive at a good approximation to a path length distribution function. The latter, in turn, is required for inferring the source composition, estimating the confinement time, and distinguishing between various models of diffusion and trapping. The elucidation of these astrophysical phenomena involves an iterative, trial-and-error procedure, but the rich variety of information contained in the observed cosmic rays will make it possible to arrive at a self-consistent picture.

Two measured flux ratios have proved especially valuable as indices of cosmic ray path lengths: the ratio of the arriving ensemble Li-Be-B to the abundant group $\mathrm{C}-\mathrm{N}-\mathrm{O}$, and the ratio of arriving ${ }^{3} \mathrm{He}$ to ${ }^{4} \mathrm{He}$. The elements $\mathrm{Li}, \mathrm{Be}$ and $\mathrm{B}$, and the isotope ${ }^{3} \mathrm{He}$ are vanishingly scarce in the general distribution of the nuclides, and there are cogent reasons to doubt that their abundance in the sources suffices to supply the fluxes observed near the earth. Hence (and also on other grounds), their presence in the cosmic ray stream is attributed to the fragmentation of heavier nuclei by collisions in space, mostly with hydrogen nuclei. (In the case of ${ }^{3} \mathrm{He}$, the parent is mainly ${ }^{4} \mathrm{He}$, and the breakup is a simple stripping process which yields ${ }^{3} \mathrm{He}$ either directly or through the production and decay of tritium.)

The result 0.25 for the abundance ratio $\mathrm{Li}+\mathrm{Be}+\mathrm{B} / \mathrm{C}+\mathrm{N}+\mathrm{O}$, measured by $\mathrm{O}$ 'Dell et al. (1962) and recently confirmed by Von Rosenvinge et al. (1969), was obtained 
for relativistic nuclei. After geomagnetic correction, the ratio outside of the Earth's magnetosphere is $0.24 \pm 0.02$. This value implies a mean path length of $4.0 \pm 1.0 \mathrm{~g} / \mathrm{cm}^{2}$ (Shapiro and Silberberg, 1967). It is noteworthy that even at energies below $100 \mathrm{MeV} /$ nucleon, Garcia-Munoz and Simpson (1970) obtained a value of $0.22 \pm 0.04$ for the foregoing ratio. At these low energies, to be sure, evaluation of path length (or of other astrophysical parameters) involves rather uncertain corrections due to solar modulation. The value $4.0 \mathrm{~g} / \mathrm{cm}^{2}$ for the path length (in the oversimplified model wherein all particles are assumed to traverse the same thickness of matter) has been confirmed independently from the isotopic composition of cosmic ray helium: ${ }^{3} \mathrm{He} /\left({ }^{3} \mathrm{He}+{ }^{4} \mathrm{He}\right.$ ) $\approx 0.1$ at energies of about $200-300 \mathrm{MeV} /$ nucleon (O'Dell et al., 1965; Ramaty and Lingenfelter, 1969).

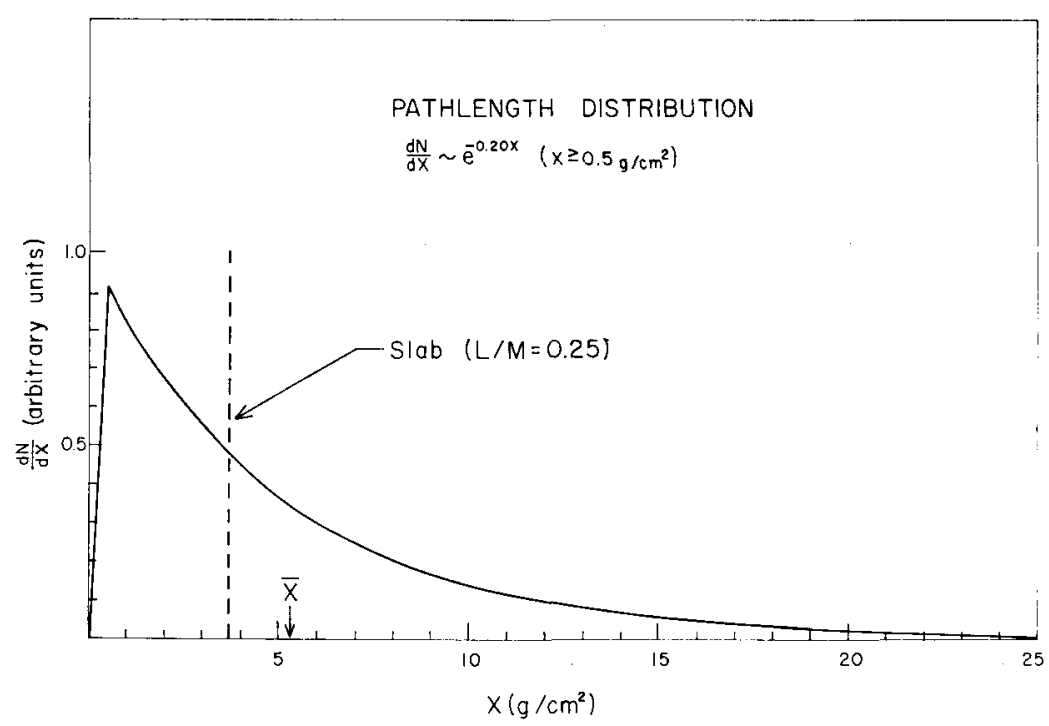

Fig. 2. Exponential-type distribution of cosmic-ray path length versus slab approximation. The former fits all the data reasonably well. The latter satisfies the observed ratio of light to medium nuclei, but it predicts an excessive production of heavier secondaries, e.g., 'sub-iron' elements from iron.

Whether from a single source or a multiplicity of sources, there must be a considerable spread in the path lengths of cosmic rays (Davis, 1959; Cowsik et al., 1967). In fact, although the 'slab' approximation satisfies the two abundance ratios discussed above, this simple model is inadequate when it is used to compute an expected production rate in space for the principal products of iron. The predicted rate based on a 'slab thickness' of $4.0 \mathrm{~g} / \mathrm{cm}^{2}$ is nearly twice the observed one. An exponential type of distribution in path length like the one in Figure 2 provides a satisfactory fit to the available experimental data (Shapiro et al., 1970b, d). [The value of the exponent $-(0.20 \pm 0.05)$ shown in Figure 2 supersedes that given in these references: the change is due to new cross-section data.] 


\section{Recent Calculations of Cosmic-Ray Transformations}

To calculate the source composition or the confinement time of galactic cosmic rays, it is necessary to solve the diffusion equations describing the collision breakup of parent nuclides, and the production of secondary ones in successive increments of path length. One must first adopt 'best' values for (a) the abundance ratios of various arriving cosmic-ray species, and (b) the partial cross-sections for many important fragmentation reactions.

The NRL group (Shapiro et al., 1970a, b, c; Shapiro and Silberberg, 1970b) started with an assumed (trial) source composition similar to that in the 'universal' abundances adopted by Cameron (1968), and fed this progenitor composition into the diffusion equations, using the exponential distribution in path length described in Section 3. They thus obtained a first-approximation composition for the arriving particles. They then adjusted the initial composition in successive approximations until the calculated abundance ratios matched those ratios for elements from carbon to iron which are reasonably well known from observations at the top of the atmosphere. With the many constraints imposed by the observed values of the elemental ratios, the calculated primordial distribution turns out to be insensitive to the initially assumed source composition.

The calculations yielded relative (arriving) abundances of 60 principal stable nuclides from ${ }^{6} \mathrm{Li}$ to ${ }^{56} \mathrm{Fe}$, and the contributions from many of the unstable ones.* Products of secondary and tertiary collisions were included. In addition, the energy dependence of partial cross-sections, the effects of ionization and collision loss, solar modulation, geomagnetic cutoff, and the shape of the cosmic-ray spectrum were taken into account. Corrections were applied for collisions with interstellar helium as well. The calculated charge distribution arriving at the earth agrees well with observed relative abundances of the individual elements ranging from $\mathrm{Li}$ to $\mathrm{Fe}$.

In the earlier NRL work, the breakup cross sections for collision with hydrogen were adopted mainly from Beck and Yiou (1968), or calculated by the methods of Audouze et al. (1967), Rudstam (1966), and Dostrovsky et al. (1968). These were revised by use of empirical data when available. Subsequently, Silberberg and Tsao (1970) devised a modified form of Rudstam's relation. Comparing their calculated results with about 300 experimental cross-section data, they found a root-mean-square error $\log \sigma_{\text {exper }}-\log \sigma_{\text {calc }} \approx 0.1$, corresponding to a standard deviation of about $25 \%$. A representative list of cross sections used in the NRL work, for fragmentation of relativistic ${ }^{12} \mathrm{C},{ }^{16} \mathrm{O},{ }^{20} \mathrm{Ne},{ }^{24} \mathrm{Mg},{ }^{28} \mathrm{Si}$ and ${ }^{56} \mathrm{Fe}$ against hydrogen, is given in Table III. More extensive sets of similar cross-sections, at energies of 150,400 , and $\geqslant 2300 \mathrm{MeV} /$ nucleon will be found in Tables XXI-XXIV of the recent review by Shapiro and Silberberg (1970b).**

\footnotetext{
* A list of these nuclides is given by Shapiro et al. (1970d), Table I. The values of abundance adopted at the top of the atmosphere (relative to C) can be found in Table II of the same paper.

** Some of our values for production of ${ }^{9} \mathrm{Be}$ and ${ }^{10} \mathrm{Be}$ [Shapiro and Silberberg (1970a)] are superseded by new values in the present Table III. The changes are due mainly to new cross-section measurements reported by Fontes et al. (1970).
} 
TABLE III

Partial cross-sections (in millibarns) for production of various nuclides form collisions of six major cosmic-ray nuclides with hydrogen; $E \geqslant 2.3 \mathrm{GeV} /$ nucleon

\begin{tabular}{|c|c|c|c|c|c|c|}
\hline \multirow[t]{2}{*}{ Product } & \multicolumn{2}{|c|}{ Target ${ }^{\mathrm{a}}$} & \multirow[b]{2}{*}{${ }^{20} \mathrm{Ne}$} & \multirow[b]{2}{*}{${ }^{24} \mathrm{Mg}$} & \multirow[b]{2}{*}{${ }^{28} \mathrm{Si}$} & \multirow[b]{2}{*}{${ }^{56} \mathrm{Fe}$} \\
\hline & ${ }^{12} \mathrm{C}$ & ${ }^{16} \mathrm{O}$ & & & & \\
\hline${ }^{6} \mathrm{Li}$ & $7^{\mathrm{b}}$ & 14 & 12 & 13 & 13 & 30 \\
\hline${ }^{7} \mathrm{Li}$ & 6 & 14 & 11 & 11 & 11 & 20 \\
\hline${ }^{7} \mathrm{Be}$ & 10 & 11 & 10 & 10 & 10 & 8.5 \\
\hline${ }^{9} \mathrm{Be}$ & 6 & 3.7 & 3 & 3 & 3 & 5 \\
\hline${ }^{10} \mathrm{Be}$ & 3.5 & 1.0 & 1.9 & 1.9 & 1.9 & 4 \\
\hline${ }^{10} \mathrm{~B}$ & 14 & 12 & 9 & 8 & 7 & 7 \\
\hline${ }^{11} \mathrm{~B}$ & 53 & 25 & 18 & 15 & 12 & 9 \\
\hline${ }^{12} \mathrm{C}$ & & 24 & 18 & 13 & 10 & 7 \\
\hline${ }^{13} \mathrm{C}$ & & 20 & 14 & 10 & 8 & 5 \\
\hline${ }^{14} \mathrm{~N}$ & & 26 & 18 & 13 & 10 & 6 \\
\hline${ }^{15} \mathrm{~N}$ & & 50 & 23 & 17 & 13 & 6 \\
\hline${ }^{16} \mathrm{O}$ & & & 24 & 18 & 13 & 6 \\
\hline${ }^{17} \mathrm{O}$ & & & 25 & 19 & 14 & 6 \\
\hline${ }^{18} \mathrm{O}$ & & & 23 & 16 & 12 & 6 \\
\hline${ }^{19} \mathrm{~F}$ & & & 45 & 19 & 14 & 7 \\
\hline $20,21,22 \mathrm{Ne}$ & & & & 69 & 55 & 21 \\
\hline${ }^{23} \mathrm{Na}$ & & & & 51 & 23 & 9 \\
\hline $24,25,26 \mathrm{Mg}$ & & & & & 77 & 25 \\
\hline${ }^{27} \mathrm{Al}$ & & & & & 52 & 10 \\
\hline$\sigma_{i}$ & $205^{c}$ & 260 & 315 & 355 & 400 & 676 \\
\hline
\end{tabular}

${ }^{a}$ In the present context, the 'target' is, of course, a cosmic-ray nucleus which collides with interstellar hydrogen.

b Italicized values refer to cross-sections based primarily upon experimental information.

c The quantity $\sigma_{i}$ is the total inelastic cross-section.

\section{Composition at the Sources}

The primordial composition of cosmic rays can provide insight into the nature of the sources, their evolution, and the processes of nucleosynthesis that occur in those regions. Following the procedures outlined in Section 4, Shapiro et al. (1970c) obtained the source composition shown in Table IV (this is a revised version of the table previously published by the same authors). The Table shows the calculated primordial abundances of the principal even- $Z$ elements, and that of nitrogen, relative to carbon. These results show that $\mathrm{C}, \mathrm{N}, \mathrm{O}, \mathrm{Ne}, \mathrm{Mg}, \mathrm{Si}$, and $\mathrm{Fe}$ are present in the sources, while one cannot yet be sure that $\mathrm{S}$ and $\mathrm{Cr}$ are primordial constituents. The abundances in Table IV can be compared with those computed by Beck and Yiou (1968), under the slab model approximation.

In Table V, all the elements listed were assumed (in the calculations) to be secondary. Unlike the primordial values in Table IV, which were adjusted to give the best fit to the arriving cosmic-ray distribution, the values in Table $\mathrm{V}$ were unadjusted. Observed abundances of these elements are shown for comparison. All data were normalized to a value of 100 for carbon. 
TABLE IV

\begin{tabular}{|c|c|c|c|c|c|c|c|c|c|}
\hline \multirow[b]{2}{*}{ Element } & \multicolumn{8}{|c|}{ Primordial abundances of cosmic rays relative to carbon } & \multirow[b]{2}{*}{$\mathrm{Fe}$} \\
\hline & $\mathrm{C}$ & $\mathbf{N}$ & $\mathrm{O}$ & $\mathrm{Ne}$ & $\mathrm{Mg}$ & Si & $\mathbf{S}$ & $\mathrm{Cr}$ & \\
\hline Relative & $100^{\mathrm{a}}$ & 12 & 102 & 21 & 27 & 23 & 4 & 4 & 23 \\
\hline abundance & & \pm 3 & \pm 6 & \pm 3 & \pm 4 & \pm 4 & \pm 2 & \pm 4 & \pm 5 \\
\hline
\end{tabular}

a Normalization.

TABLE V

Abundances of some secondary ${ }^{a}$ cosmic-ray elements at the top of the atmosphereb

\begin{tabular}{lrrrrrrrrrrr}
\hline Element & $\mathrm{Li}$ & $\mathrm{Be}$ & $\mathrm{B}$ & $\mathrm{F}$ & $\mathrm{P}$ & $\mathrm{Cl}$ & $\mathrm{K}$ & $\mathrm{Sc}$ & $\mathrm{Ti}$ & $\mathrm{V}$ \\
\hline Calculated & 18 & 12 & 25 & 2.9 & 0.6 & 0.6 & 0.8 & 0.4 & 1.7 & 0.9 \\
abundance & \pm 2 & \pm 1 & \pm 3 & \pm 0.7 & \pm 0.2 & \pm 0.3 & \pm 0.3 & \pm 0.2 & \pm 0.7 & \pm 0.3 \\
& & & & & & & & & & \\
Observed & 16 & 11 & 27 & 2 & $\mathbf{0 . 6}$ & 0.5 & 0.6 & 0.3 & 2 & 1.0 \\
abundance & \pm 2 & \pm 3 & \pm 3 & \pm 1 & +1.4 & \pm 0.3 & \pm 0.3 & \pm 0.2 & \pm 0.5 & \pm 0.3 \\
& & & & & -0.5 & & & & &
\end{tabular}

a Assumed to be absent from the primordial flux for purposes of calculation.

b All normalized to a value of 100 for carbon.

Figure 3 compares the calculated abundances of cosmic-rays at the sources with those observed near the Earth for kinetic energies $>1.5 \mathrm{GeV} /$ nucleon, all normalized to the observed flux of carbon.* In each pair of adjacent columns, the one at the right (diagonally hatched) represents the amount of the primordial element that would have arrived if it had not interacted with interstellar matter. The left column shows the abundance observed at the top of the atmosphere, with the blank lower section representing the surviving primordial fraction, while the upper dark area indicates the relative contribution of secondary products.

It is seen that primordial oxygen slightly exceeds carbon, rather than vice versa as observed near the Earth. Nitrogen is relatively less abundant at the sources than in the arriving flux, and in the latter, it is largely secondary. Of the primordial cosmicray elements heavier than oxygen, the principal ones are neon, magnesium, silicon, and iron. These, along with carbon and oxygen, are attenuated while penetrating the interstellar medium. Owing to its large cross-section for fragmentation, appreciably more than half of the initial ${ }^{56} \mathrm{Fe}$ breaks up into lighter species, among them ${ }^{55} \mathrm{Fe}$, and less than $30 \%$ of the primordial iron arrives at the Earth. Some $90 \%$ of the arriving $\mathrm{O}$, and $\mathrm{Fe}$, and roughly $70-80 \%$ of the incident $\mathrm{C}, \mathrm{Ne}, \mathrm{Mg}$ and $\mathrm{Si}$ are primordial, while secondaries predominate among the other elements between Li and

* This figure is a revised version of a similar one previously published by the NRL group. 


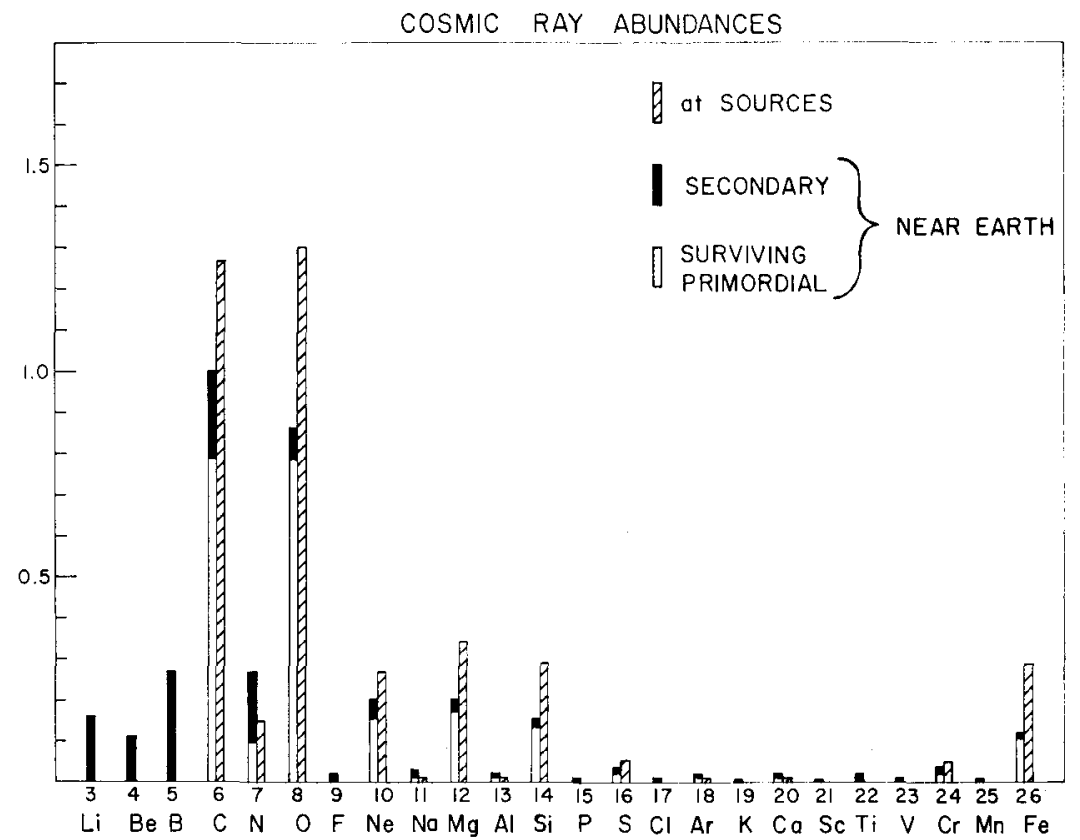

Fig. 3. Relative abundances of primordial cosmic-rays (diagonally hatched), and those observed near the Earth. The dark protion of each (left) column represents the contribution of secondary nuclei generated en route.

Fe. There seem to be traces of $\mathrm{Na}, \mathrm{Al}, \mathrm{Ar}$ and $\mathrm{Ca}$ at the sources, but this is not yet firmly established.

Figure 4 compares the source composition of cosmic rays with the makeup of a well-defined sample of stellar matter, i.e., the Sun's photosphere (Aller, 1961; Unsöld, 1969). In this diagram, the higher solar abundance of Fe labeled ' 2 ' reflects the recent analysis of Garz et al. (1969), which has brought photospheric Fe into reasonable agreement with coronal $\mathrm{Fe}$, and with the meteoritic ratios $\mathrm{Fe} / \mathrm{Si}$ and $\mathrm{Fe} / \mathrm{Mg}$. Not shown in Figure 4 are the source abundances of hydrogen and helium. These are known only crudely, and relative to primordial carbon, they are deficient by a factor of $\sim 10$ to 25 in the cosmic-ray sources as compared with the Sun. Conversely, $\mathrm{Mg}, \mathrm{Si}$ and $\mathrm{Fe}$ are disproportionately plentiful in the primordial cosmic-ray distribution. Accordingly, the ratio of cosmic-ray source abundance to solar abundance is greater by nearly two orders of magnitude for $\mathrm{Mg}$, $\mathrm{Si}$, or $\mathrm{Fe}$ than it is for hydrogen and helium. A similar comparison could be made with the composition of stars in Population I generally. This salient feature of the cosmic-ray composition is now established securely, in the light of the improved cosmic-ray and cross-sectional data, that warranted more refined diffusion calculations. However, the relative preponderance of heavier elements in the cosmic rays was already noticed some years ago (Hayakawa, 1956; Ginzburg, 1958; Aizu et al., 1960; Shapiro, 1962), and it has long been an argument for cosmic-ray origin in the sites of supernova explosions. In addition, 


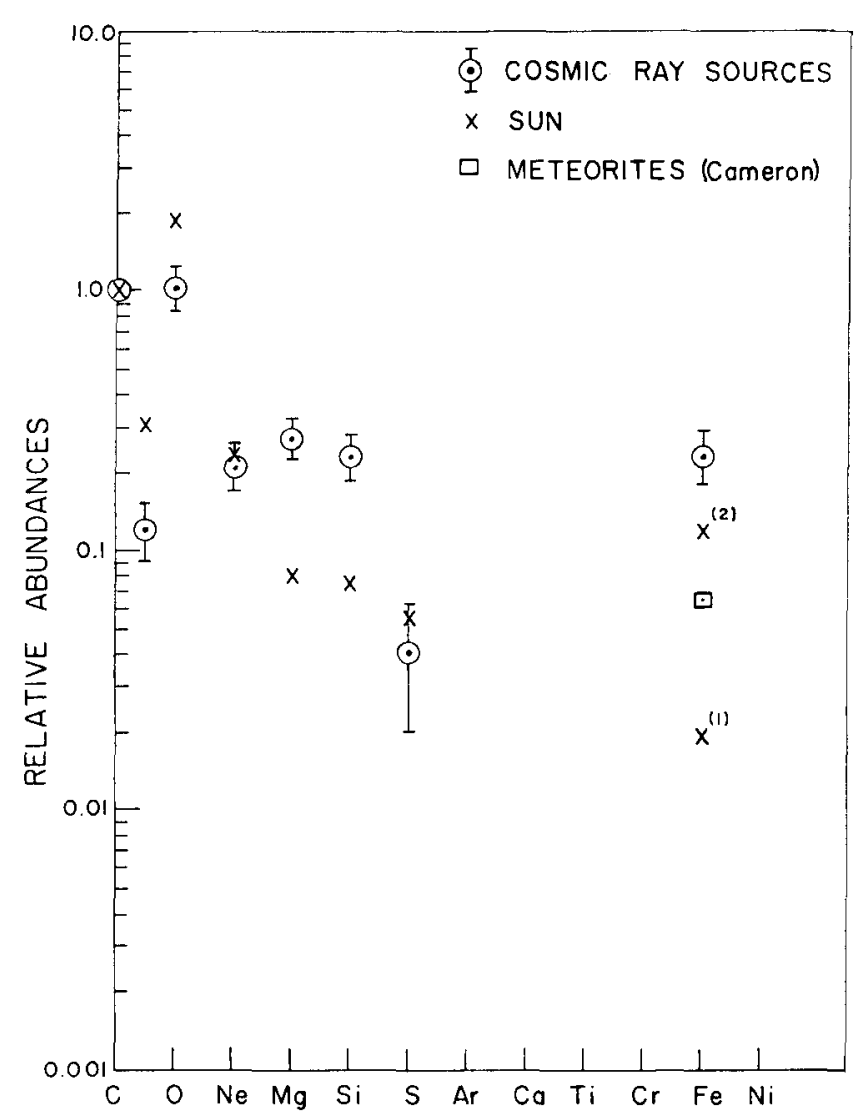

Fig. 4. Cosmic-ray abundances calculated at the sources versus composition of the solar photosphere. All data have been normalized with respect to carbon. Both the old value for photospheric iron (labeled 1), and the new value (2) are plotted.

the nucleogenetic schemes devised by Burbidge et al. (1957), e.g., the ' $r$-process' of successive neutron capture, result in the rapid build-up of heavier elements in such stellar explosions, and this bolsters the supernova hypothesis.

More recent work on the explosive nuclear burning of carbon, oxygen and silicon in evolved massive stars (Arnett and Clayton, 1970) points the way to possible alternative means of producing heavier elements in a cosmic-ray-like distribution. The discovery of pulsars in the remnants of supernovae, and their interpretation as neutron stars having enormous rotational energy (Gold, 1969) has solved, at least in principle, the mystery of the powerful source required for continuous replenishment of the high energy electrons in objects like the Crab Nebula. We still have far to go before a satisfactory theory of cosmic-ray genesis will have been achieved. But it seems possible at least to conclude, in the light of the findings summarized above on primordial composition, that most, if not all, of the cosmic-rays have their origin in highly evolved stars in which nucleosynthesis has reached an advanced stage. 


\section{Confinement and Lifetime}

We have seen how the transformations produced by nuclear collisions of cosmic rays in space help us to reconstruct their distribution in path length and their primordial composition. Some of these nuclear transmutations can also clarify other aspects of their propagation, such as their mean storage time $T$, and the region(s) of their confinement.

If we knew the mean density $\varrho$ of gas in the storage region(s) - or, better, the density distribution - then we could compute the mean lifetime from the distribution in path length. For illustration, consider the simple approximation in which all the particles pass through a uniform thickness $\lambda$. Then

$$
\lambda=\varrho \beta c T .
$$

With $\lambda=4 \mathrm{~g} / \mathrm{cm}^{2}, \beta=1$ (for relativistic particles), and $\varrho=1.6 \times 10^{-24} \mathrm{~g} / \mathrm{cm}^{3}$ ( 1 hydrogen atom per $\mathrm{cm}^{3}$ ) for the mean interstellar density in the galactic disk, we find

$$
T=8 \times 10^{13} \mathrm{sec}=2.5 \times 10^{6} \mathrm{yr} .
$$

Such an estimate of the storage lifetime is based on the implicit assumption that the cosmic rays are confined to the disk of the Galaxy. This is a plausible model in the light of the dynamical properties of the cosmic-rays gas (Parker, 1968), as well as the tight magnetic confinement of most individual cosmic-ray particles. Thus, some $99.99 \%$ of cosmic-ray nuclei have energies $<10^{12} \mathrm{eV} /$ nucleon, and Larmor radii $<10^{-3} \mathrm{pc}$ in the galactic magnetic fields. When these modest gyro-radii are compared with the disk thickness of several hundred $\mathrm{pc}$, it is understandable that the galactic magnetic fields ( $\approx$ several micro-gauss) can trap the cosmic rays for long times, notwithstanding their high momenta. Indeed, their observed isotropy attests to efficient processes of stirring and storage.

Nevertheless, we cannot yet dismiss the possibility that a significant fraction of the cosmic rays might be circulating freely between the disk and the neighboring region the galactic halo. There the mean density is thought to be $\lesssim 10^{-2}$ of the disk density, and the magnetic fields considerably weaker than those near the galactic plane. Hence a cosmic-ray nucleus might survive 100 times as long, perhaps $\approx 10^{8} \mathrm{yr}$, in the halo. Accordingly, if we had an independent method of deducing the mean confinement time, and this yielded a value $T \approx 10^{8} \mathrm{yr}$, we could infer that the cosmic rays observed near the earth spend a major part of their lifetime in the halo. Conversely, if we found $T \ll 10^{8} \mathrm{yr}$, this would further bolster the case for confinement in the disk.

Some years ago it was proposed by Hayakawa et al. (1958) and by Peters (1963) that one of the secondary nuclides produced by fragmentation in space could serve as a useful radioactive 'clock' for estimating cosmic-ray lifetime. As we have seen, the total flux of $\mathrm{Li}, \mathrm{Be}$, and $\mathrm{B}$ that arrives at the Earth depends on the amount of material they have traversed. However, the flux of $\mathrm{Be}$ relative to that of $\mathrm{B}$, or of $\mathrm{Be} / \mathrm{Li}$ depends on the length of time that the particles have been traveling, together with their progeny. In particular, one of the collision products, ${ }^{10} \mathrm{Be}$, decays with a mean life $\tau_{0}$ 
(at rest) of 3,9 million years into ${ }^{10} \mathrm{~B}$, and it has a range of energy-dependent lifetimes $\tau=\gamma \tau_{0}$ owing to relativistic time dilation ( $\gamma$ is the Lorentz factor). Considering the production and decay of the various $B e$ and $B$ isotopes all along their path, the relative amounts of elemental Be and B change as a function of time. For times short compared to the ${ }^{10} \mathrm{Be}$ lifetime, most of this nuclide will have survived, and the amount of $B$ present will depend mainly upon the direct production of the B (or such precursors as ${ }^{11} \mathrm{C}$ ) by fragmentation. However, if the cosmic rays survive for times long compared with $\tau$, then the ${ }^{10} \mathrm{Be}$ will have largely disappeared, having been converted to ${ }^{10} \mathrm{~B}$. The ratio of elemental $\mathrm{Be}$ to elemental $\mathrm{B}$ arriving at the earth will then approach some smaller limiting value.

Several years ago Daniel and Durgaprasad (1966) applied this method, and concluded that $T \gtrsim 50 \times 10^{6} \mathrm{yr}$. Shapiro and Silberberg (1967) analyzed the method, and found that this lower limit might be subject to drastic revision, in view of uncertainties in cross sections and the paucity of data on the pertinent abundances. They took note of the cross-section measurements of Bernas et al. (1967), Gradsztajn et al. (1965), Gradsztajn (1967), and Yiou et al. (1968), and concluded that a much shorter cosmicray age, say 1-10 million years, could not yet be ruled out. Subsequently, Von Rosenvinge et al. (1969) measured the ratios $\mathrm{Be} / \mathrm{B}$ and $\mathrm{Be} / \mathrm{Li}$ with a counter telescope, and obtained results of great statistical weight. Meanwhile, Shapiro and Silberberg have re-calculated the expected light-element ratios, using the latest cross-section data (Yiou et al., 1969; Fontes et al., 1970). Their new cosmic-ray compilation of emulsion data on light-element abundances agree with the results of Von Rosenvinge et al. (1969).

A comparison of the calculated values of $\mathrm{Be} /(\mathrm{Li}+\mathrm{B})$ (as a function of energy per nucleon) with available data on relative abundances of the arriving nuclides is shown in Figure 5. The upper curve was computed for the asymptotic case $T \ll \tau$; this permits survival of the $\mathrm{Be}^{10}$ and a "high" ratio of $\mathrm{Be} /(\mathrm{Li}+\mathrm{B})$ in the arriving cosmic rays. The lower curve corresponds to $T \gg \tau$, which implies that practically all of the $\mathrm{Be}^{10}$ has decayed en route. The data points represent observational results: $E$, emulsion experiments $W_{b}$, counter experiments by Webber's group at balloon altitudes (Von Rosenvinge et al., 1969); and, at the lowest energies, C+G, observations from satellites and space probes by Simpson's group in Chicago (Fan et al., 1968; Comstock et al., 1969; Garcia-Munoz and Simpson, 1970) and McDonald's group at the Goddard Space Flight Center (Balasubrahmanyan et al., 1966; Hagge et al., 1968). The data points labeled $W_{s}$ were obtained more recently by Webber's group (Lezniak et al., 1970) in the Pioneer 8 satellite.

The abrupt break in the middle of each curve (at about $2 \mathrm{GeV} /$ nucleon) is artificial; it results from our preference to incorporate corrections for geomagnetic distortion of composition in the calculated ratios rather than in the experimental data. In the left part of the diagram, the dashed curves represent computed values of $\mathrm{Be} /(\mathrm{Li}+\mathrm{B})$ outside of the Earth's magnetosphere without correction for rigidity cutoff. These curves may be fairly compared with the experimental results plotted at kinetic energies $<2 \mathrm{GeV} /$ nucleon, since the latter were obtained outside of the magnetosphere, or 


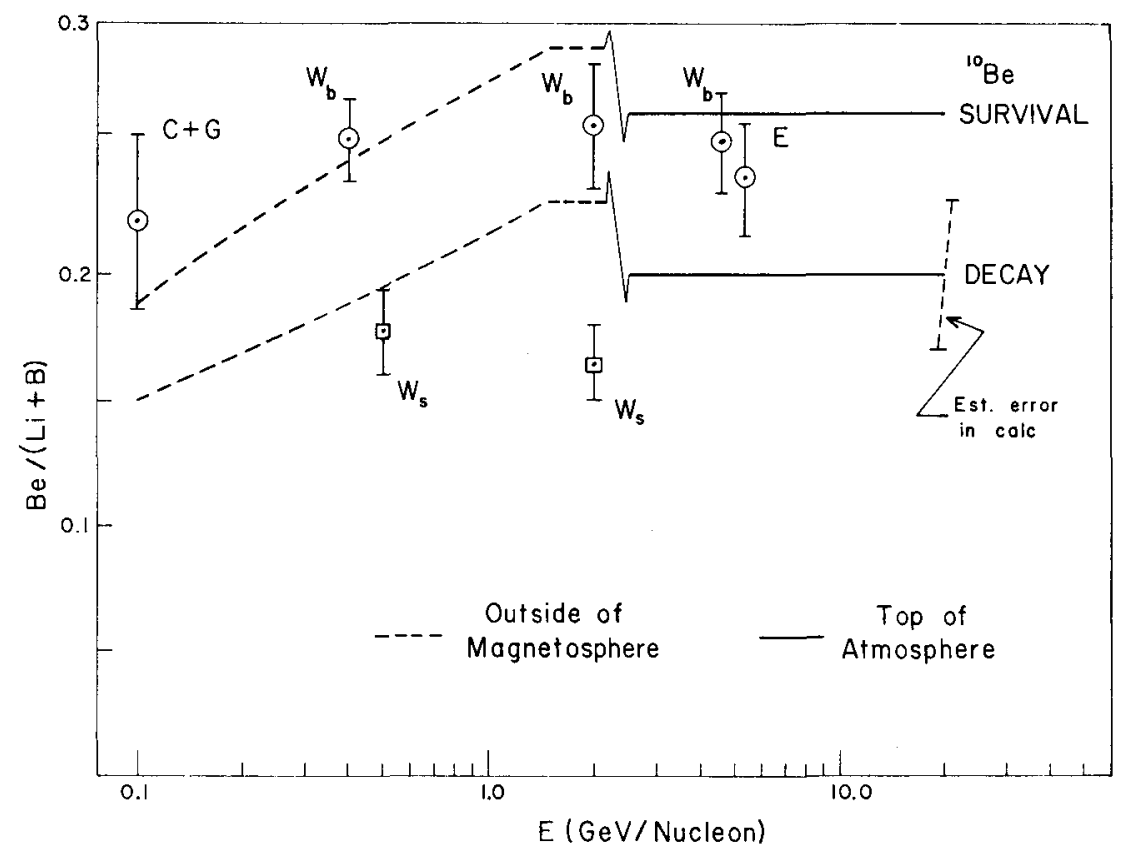

Fig. 5. Observed values of the abundance ratio $\mathrm{Be} /(\mathrm{Li}+\mathrm{B})$ versus energy/nucleon, and those calculated for the asymptotic cases of ${ }^{10} \mathrm{Be}$ survival and decay. The solid curves at the right have been corrected from the top of the magnetosphere down to the top of the atmosphere to permit comparison with observed data that were subject to rigidity cutoff (see text).

in balloon flights at Ft. Churchill, where a rigidity cutoff did not affect the measured abundances under the conditions of the experiment. The solid curves at the right, at energies $>2 \mathrm{GeV} /$ nucleon represent calculated ratios which were corrected for modulation of the incoming relative abundances by the Earth's magnetic field. This was required for meaningful comparison with data plotted here, which were subject to rigidity cutoff.* (If the solid curves were not adjusted for passage through the magnetosphere, then these curves would connect smoothly with the dashed curve. In that case, the observational data would have required correction to the outside of the magnetosphere, and their positions relative to the calculated curves would have been the same.)

As indicated in Figure 5, the errors in both the calculated and observed abundance ratios are sizable. (Moreover, there are conflicting data at intermediate energies, which will be discussed below.) Yet a comparison of the data points labeled by circles with the upper and lower curves favors substantial ${ }^{10} \mathrm{Be}$ survival rather than decay. This would imply that the cosmic-ray age does not exceed 50 million years, and would

* The depression of these curves (at the right) is due mainly to the discrimination of the Earth's magnetic field against the important isotope ${ }^{7} \mathrm{Be}$, with its low ratio of mass to charge. (For a discussion of ${ }^{7} \mathrm{Be}$ in the cosmic rays, see Shapiro and Silberberg $(1968,1970 \mathrm{~b})$ ). The geomagnetic modulation of the relative isotopic and elemental abundances is treated in the Appendix of the latter paper.) 
suggest that it is probably $<20$ million years. Such a lifetime would be more nearly consistent with confinement of the cosmic-rays mainly in the galactic disk rather than in the larger volume of the halo.*

However, the evidence for this point of view must be considered very inconclusive, especially in the light of the satellite data labeled with squares $\left(W_{s}\right)$ in Figure 5 . These data taken alone would lead to an opposite conclusion, in favor of ${ }^{10} \mathrm{Be}$ decay, and a lifetime exceeding $10^{8} \mathrm{yr}$. The contradiction between the points $W_{b}$ and $W_{s}$ is very puzzling; i.e., Webber's balloon observations and the satellite observations reported by his group seem irreconcilable.**

\section{Concluding Remarks}

An excellent start has been made in detecting and identifying the ultra-heavy cosmicray elements $(Z>30)$ which are very scarce indeed, collectively $\sim 10^{-4}$ of the iron abundance (Fowler et al., 1970). This nuclear component, extending up to $Z \approx 90$ and perhaps even to transuranic elements, will provide critical data on source composition and on confinement (Mewaldt et al., 1970).

The study of isotopic abundances for cosmic-ray elements with $Z \geqslant 3$ is only in its infancy. As techniques improve in the coming years, this study will surely contribute much to our knowledge of sources and of propagation.

In conclusion, it seems plain that much arduous work remains in tapping the wealth of information contained in the detailed composition - both elemental and isotopic of the cosmic rays.

The author is grateful to his colleagues, Dr R. Silberberg and Dr C. H. Tsao for valuable advice and assistance.

\section{References ${ }^{\dagger}$}

Aizu, H., Fujimoto, Y., Hasegawa, S., Koshiba, M., Mito, I., Nishimura, J., and Yokoi, K.: 1960, Suppl. Progr. Theoret. Phys. 16, p. 54ff.

Aller, L. H.: 1961, The Abundance of Elements, Interscience Publishers, Inc., New York.

Arnett, W. D. and Clayton, D. D.: 1970, Nature 227, 780.

Audouze, J., Epherre, M., and Reeves, H.: 1967, Nucl. Phys. A97, 144.

Balasubrahmanyan, V. K., Hagge, D. E., Ludwig, G. H., and McDonald, F. B.: 1966, J. Geophys. Res. 71, 1771.

Beck, F. and Yiou, F.: 1968, Astrophys. Letters 1, 75.

Bernas, R., Gradsztajn, E., Reeves, H., and Schatzman, E.: 1967, Ann. Phys. 44, 425.

Burbidge, E. M., Burbidge, G. R., Fowler, W. A., and Hoyle, F.: 1957, Rev. Mod. Phys. 29, 547.

Cameron, A. G. W.: 1968, in Origin and Distribution of the Elements (ed. by L. H. Ahrens), Pergamon Press, London, New York, p. 125ff.

* It is noteworthy that proponents of the halo hypothesis are taking a fresh look at the alternative, the disk model (see, e.g., Ginzburg, 1970).

** Further experimental support for the curve of survival comes from the recent Be/B result of Corydon-Petersen et al. (1970), who used a new type of detector telescope.

+ A more extensive bibliography of pertinent references appears in a review by Shapiro and Silberberg (1970b). 
Clark, G.: 1970, 'Survey of New Results in Gamma-Ray Astronomy', in 'New Techniques in Space Astronomy', IAU Symp. 41, August 10-14, 1970, Munich (to be published).

Clark, G. W., Garmire, G. P., and Kraushaar, W. L.: 1968, Astrophys. J. 153, L203.

Comstock, G. M., Fan, C. Y., and Simpson, J. A.: 1969, Astrophys. J. 155, 609.

Corydon-Petersen, O., Dayton, B., Lund, N., Melgaard, K., Omø, K., Peters, B., and Risbo, T.: 1970, Nucl. Instr. Methods 81, 1.

Cowsik, R., Pal, Y., Tandon, S. N., and Verma, R. P.: 1967, Phys. Rev. 158, 1238.

Crouch, M. F., Curr, H. S., Kropp, W. R., Lathrop, J., Meyer, B. S., Reines, F., Sellschop, J. P. F., and Sobel, H. W.: 1970, Proc. 11th Int. Conf. on Cosmic Rays, Paper MU 21, 'Deep Underground Meaurement of Cosmic Ray Muons and Neutrinos', (to be published in Acta Phys. Hung.).

Daniel, R. R. and Durgaprasad, N.: 1966, Progr. Theor. Phys. 35, 36.

Daniel, R. R. and Stephens, S. A.: 1970, Space Sci. Rev. 10, 599.

Davis, L., Jr.: 1959, Proc. Moscow Cosmic Ray Conf. (IUPAP) 3, 220.

Dostrovsky, I., Gauvin, H., and Lefort, M.: 1968, Phys. Rev. 169, 836.

Duthie, J. G.: 1968, Can. J. Phys. 46, S401.

Fan, C. Y., Gloeckler, G., and Simpson, J. A.: 1968, Can. J. Phys. 46, S549.

Fazio, G. G.: 1967, Ann. Rev. Astron. Astrophys. 5, 481.

Fichtel, C. C.: 1970, IAU Symp. 41 on 'New Techniques in Space Astronomy', Munich, (NASA preprint X-662-70-346).

Fontes, P., Perron, C., Lestringuez, J., Yiou, F., and Bernas, R.: 1970, Mass Spectrometric Measurement of Lithium and Beryllium Production Cross-Sections in ${ }^{12} C$ Spallation by High Energy Protons and $\alpha$-Particles (in press).

Fowler, P. H., Clapham, V. M., Cowen, V. G., Kidd, J. M., and Moses, R. T.: 1970, Proc. Roy. Soc. London 318, 1.

Garmire, G. and Kraushaar, W.: 1965, Space Sci. Rev. 4, 123.

Garcia-Munoz, M. and Simpson, J. A.: 1970, Acta Phys. Hung. 29, 325.

Garz, T., Holweger, H., Kock, M., and Richter, J.: 1969, Astron. Astrophys. 2, 446.

Ginzburg, V. L.: 1958, Progr. Elem. Particle Cosmic Ray Phys. 4, 339 (ed. by J. G. Wilson and S. A. Wouthuysen), North Holland Publ. Co., Amsterdam.

Ginzburg, V. L.: 1970, Comm. Astrophys. Space Phys. 2, 43.

Ginzburg, V. L. and Syrovatskii, S. J.: 1964, The Origin of Cosmic Rays, Pergamon Press, The Macmillan Co., New York.

Gold, T.: 1969, Nature 221, 25.

Gould, R. J. and Burbidge, G, R.: 1967 Hdb. Physik XLVI/2, 265 (ed. by S. Kitte and S. Flügge), Springer-Verlag, Berlin.

Gradsztajn, E.: 1967, in High-Energy Nuclear Reactions in Astrophysics (ed. by B. S. P. Shen), W. A. Benjamin Inc., New York, p. 247ff.

Gradsztajn, E., Yiou, F., Klapisch, R., and Bernas, R.: 1965, Phys. Rev. Letters 14, 436.

Greisen, K.: 1966, 'Experimental Gamma-Ray Astronomy', in Perspectives in Modern Physics, Interscience Publishers, New York, p. 355ff.

Greisen, K.: 1970, 'The Physics of Cosmic X-Ray, Gamma-Ray, and Particle Sources', in Brandeis University Summer Institute Lectures, Gordon and Breach, New York (in press).

Hagge, D. E., Balasubrahmanyan, V. K., and McDonald, F. B.: 1968, Can. J. Phys. 46, S539

Hayakawa, S.: 1956, Progr. Theoret. Phys. 15, 111.

Hayakawa, S.: 1969, Cosmic Ray Physics (ed. by R. E. Marshak), Interscience Monographs, WileyInterscience, New York.

Hayakawa, S., Ito, K., and Terashima, Y.: 1958, Progr. Theoret. Phys. Suppl. (Kyoto), 6, 1.

Krishnaswamy, S., Menon, M. G. K., Narasimham, V. S., Ito, N., Kino, S., Miyake, S., Craig, R., Parsons, A. J., and Wolfendale, A.W.: 1970, Proc. 11th Int. Conf. on Cosmic Rays, Paper MU 20 , 'The Kolar Gold Field Neutrino Experiment', (To be published in Acta Phys. Hung.).

Lezniak, J. A., Von Rosenvinge, T. T., and Webber, W. R.: 1970, Acta Phys. Hung. 29, Suppl. 1, 375.

Lüst, R. and Pinkau, K.: 1967 'Theoretical Aspects of Celestial Gamma-Rays', in Electromagnetic Radiation in Space, (ed. by J. G. Emming), Springer-Verlag, New York, 9, 231.

Mewaldt, R. A., Turner, R. E., Friedlander, M. W., and Israel, M. H.: 1970, Acta Phys. Hung. 29, Suppl. 1, p. 433ff.

Meyer, P.: 1969, Ann. Rev. Astron. Astrophys. 7, 1.

O'Dell, F. W., Shapiro, M. M., and Stiller, B.: 1962, J. Phys. Soc. Japan Suppl. S. A-III 17, 23. 
O'Dell, F. W., Shapiro, M. M., Silberberg, R., and Stiller, B.: 1965, Proc. 9th Int. Conf. on Cosmic Rays 1, Publ. by Inst. of Physics and the Physical Society of London, p. $412 \mathrm{ff}$.

Parker, E. N.: 1968, in Nebulae and Interstellar Matter (ed. by B. M. Middlehurst and L. H. Aller), Chapt. 14, Univ. of Chicago Press, Chicago.

Peters, B.: 1963, 'Le problème du rayonnement cosmique dans l'espace interplanétaire', in Pontificiae Academiae Scientiarum, Scripta Varia 25, 1.

Ramaty, R. and Lingenfelter, R. E.: 1969, Astrophys. J. 155, 587.

Rudstam, G.: 1966, Z. Naturf. 21a, 1027.

Shapiro, M. M.: 1962, Science 135, 175.

Shapiro, M. M. and Silberberg, R.: 1967 in High-Energy Nuclear Reactions in Astrophysics (ed. by B. S. P. Shen) W. A. Benjamin Inc., New York, Chap. 2, p. 37.

Shapiro, M. M. and Silberberg, R.: 1968, Can. J. Phys. 46, Suppl. S561.

Shapiro, M. M. and Silberberg, R.: 1970a, Acta Phys. Hung. 29, Suppl. 1, p. 485ff,

Shapiro, M. M. and Silberberg, R.: 1970b, 'Heavy Cosmic-Ray Nuclei', in Annual Review of Nuclear Science, Ann. Rev. 20, 323, Palo Alto, Calif.

Shapiro, M. M., Silberberg, R., and Tsao, C. H.: 1970a, Acta Phys. Hung. 29, Suppl. 1, p. $463 f f$.

Shapiro, M. M., Silberberg, R., and Tsao, C. H.: 1970b, Acta Phys. Hung. 29, Suppl. 1, p. $471 \mathrm{ff}$.

Shapiro, M. M., Silberberg, R., and Tsao, C. H.: 1970c, Acta Phys. Hung. 29, Suppl. 1, p. $479 f f$.

Shapiro, M. M., Silberberg, R., and Tsao, C. H.: 1970d, 'Diffusion of Cosmic Rays and Their Source Composition', in George Gamow Memorial Volume (ed. by F. Reines), Univ. of Colorado Press, Boulder (in press).

Silberberg, R. and Tsao, C. H.: 1970, Preliminary Report in Bull. Am. Phys. Soc. II 15, 618. A detailed paper will shortly be published.

Stecker, F. W.: 1971, Cosmic Gamma Rays, U. S. Government Printing Office, Washington, D.C. (in press).

Unsöld, A. O. J.: 1969, Science 163, 1015.

Von Rosenvinge, T. T., Ormes, J. F., and Webber, W. R.: 1969, Astrophys. Space Sci. 3, 80.

Yiou, F., Baril, M., Dufaure de Citres, J., Fontes, P., Gradsztajn, E., and Bernas, R.: 1968, Phys. Rev. 166, 968.

Yiou, F., Seide, C., and Bernas, R.: 1969, J. Geophys. Res. 74, 2447.

\section{General Bibliography}

Creutz, E. (ed.): 1958, 'Nuclear Instrumentation', in Hdb. Physik 45, Springer-Verlag, Berlin.

Fluegge, S. and Sitte, K. (eds.),: 1967, 'Cosmic Rays', in Hdb. Physik, 46/2, Springer-Verlag, Berlin.

Ginzburg, V. L. and Syrovatskii, S. J.: 1964, The Origin of Cosmic Rays, Pergamon Press, The Macmillan Co., New York.

Hayakawa, S.: 1969, Cosmic Ray Physics (ed. by R. E. Marshak), Interscience Monographs, WileyInterscience, New York.

Meyer, P.: 1969, Ann. Rev. Astron. Astrophys. 7, 1.

Morrison, P.: 1959, 'Cosmic Rays', in Hdb. Physik, 46/1, 1, Springer-Verlag, Berlin.

Pal. Y.: 1967, Hdb. Physics, Chapter 11 (ed. by E. U. Condon and H. Odishaw), McGraw Hill, New York.

Parker, E. N.: 1969, Space Sci. Rev. 9, 651.

Peters, B.: 1963 (ed.), Proc. of Int. School of Physics 'Enrico Fermi', Course XIX, Cosmic Rays, Solar Particles and Space Research, Academic Press, New York and London.

Proc. 9th Int. Conf. on Cosmic Rays, London: 1965, in 2 volumes, Publ. by Inst. of Physics and the Physical Society.

Proc. 10th Int. Conf. on Cosmic Rays, Calgary: 1968, Can. J. Phys. 46, Parts 2, 3, and 4. (For Origin and Galactic Phenomena, see Part 3.)

Sandström, A. E.: 1965, Cosmic Ray Physics, North-Holland Publ. Co., Amsterdam, and John Wiley \& Sons, Inc., New York.

Shapiro, M. M.: 1963, 'Notes on Cosmic Radiation', Proc. of Int. School of Physics 'Enrico Fermi', Course XXVIII, Star Evolution (ed. by L. Gratton), Academic Press, New York and London, p. $280 \mathrm{ff}$.

Shapiro, M. M.: 1968, 'Cosmic-Ray Nuclei', in Astronautics and Aeronautics, Reviews of Space Science (July), p. $6 \mathrm{ff}$. 
Shapiro, M. M. and Silberberg, R.: 1970, 'Heavy Cosmic-Ray Nuclei', in Ann. Rev. Nucl. Sci. 20, Annual Reviews, Inc., Palo Alto, Calif., p. $323 \mathrm{ff}$.

Wilson, J. G.: 1952-56 (ed.), Prog. in Cosmic-Ray Phys. I-III, North Holland Publ. Co., Amsterdam, and Interscience Publ., New York.

Wilson, J. G. and Wouthuysen, S. A.: 1958-67 (eds.), Prog. Elem. Particle Cosmic-Ray Phys. IV-IX, North-Holland Publ. Co., Amsterdam, and Interscience Division, John Wiley \& Sons, Inc., New York.

Shklovsky, I. S.: 1960, Cosmic Radio Waves, Harvard University Press, Cambridge, Mass.

Shklovsky, I. S.: 1968, Supernovae (ed. by R. E. Marshak), Interscience Monographs, Wiley-Interscience, New York.

Somogyi, A.: 1970 (ed.), Proc. IIth Int. Conf. on Cosmic Rays, 1969, Budapest, Hungary, Acta Phys. Hung. 29, Suppl. 1.

Waddington, C. J.: 1970, Some Remarks on the Composition of the Cosmic Radiation, Sixth Interamerican Seminar on Cosmic Rays. La Paz (Preprint CR-150 Univ. of Minnesota). 